\title{
Computer-Aided Diagnosis of Mammography Cancer
}

\author{
Loai Kinani, Umar Alqasemi
}

\begin{abstract}
In this study, computer-aided detection (CADe) system is optimized to reduce radiologists' workload and to improve accuracy of cancer detection by providing more quantitative (objective) decisions added to the qualitative (subjective) assessment of radiologists. The images have been collected from MIAS database. 3 databases were prepared by 3 different ROIs sizes (32x32, 42x42 \& 52x52 pixels). Then, prepressing is done to enhance the peripheral of ROIs. This CADe computed parametric features from ROIs using statistics, histogram, GLCM and wavelet techniques. Sequential Forward Selection (SFS) technique is used to study the significance of features and eventually to omit redundancies. Several types of K-Nearest Neighbor (KNN) and Support Vector Machine (SVM) classifiers were trained to differentiate between normal and abnormal ROIs, then tested on another non-training set. Best overall performance results obtained with $\mathrm{ROI}$ size of $32 \times 32$ and histogram of 32 levels (Accuracy $=97.37 \%$, Sensitivity $=95 \%$, Specificity $=100 \%, P P V=100 \%$ and $N P V=94.74)$. The results also indicate some useful features are well-representing to abnormalities across different classifiers such as: Mean, STD, Square of STD, Mode, Median, Quantile (10\%), Quantile (70\%), Quantile (90\%), Percentile (30\%), throughout multiple histogram levels both in spatial and DWT spaces.
\end{abstract}

Keywords: Mammogram, Mass, Microcalcification, computer-aided detection, medical image recognition, support vector machine.

\section{INTRODUCTION}

B reast cancer is the most common cause of death from cancer family in women worldwide. Early stage detection, and treatment plays important rule in decreasing mortality rate. Digital imaging or mammography is commonly for breast mass and microcalcifications (MCCs) screening and diagnosis [1]. MCCs are the main findings that point out to possibility of cancer in the breast tissue at the early stage. Mass is region which has highly dense texture compared to surrounding breast tissue, and it is described by the shape, size and location.

Revised Manuscript Received on June 15, 2020.

* Correspondence Author

Loai Kinani*, Graduate Student of M.Sc. Program of Biomedical Engineering, Dept. of Electrical and Computer Engineering, King Abdulaziz University, P.O. Box 80200, Jeddah 21589, Saudi Arabia

Umar Alqasemi, Associate Professor of Biomedical Engineering, Dept. of Electrical and Computer Engineering, King Abdulaziz University, P.O. Box 80200, Jeddah 21589, Saudi Arabia

(C) The Authors. Published by Blue Eyes Intelligence Engineering and Sciences Publication (BEIESP). This is an open access article under the CC BY-NC-ND license (http://creativecommons.org/licenses/by-nc-nd/4.0/)
One the other hand, macrocalcifications are accumulations of calcium in lymphatic vessel where they usually block these vessels in the breast tissue, which causes accumulation of wastes, and chemical imbalance, opening the door for infections, elevated immune/autoimmune response, poor tissue healing that may lead eventually to birth of cancer cells.

In addition to diagnosis techniques used by radiologists, computer-aided design (CAD) systems provide a secondary check point in detecting/affirming abnormalities, and assist in making diagnostic decisions. CAD systems are computerized algorithms developed using machine-learning techniques, specifically, algorithms similar to the ones used in artificial intelligence and face/pattern/letter recognition and data mining. For such algorithms, a prior data is required to train classifiers for them to be used on future data[2]. In CAD systems, various algorithms are used to analyze medical images and give a response to aid the radiologist. In general, there are two types of responses and, consequently, two types of CAD systems. In CADe (computer-aided detection) systems, the general aim of the system is to detect abnormalities in medical images regardless of their type of abnormality. Therefore, the output of a CADe system is binary (abnormal or normal). CADe system can be used to reduce the number of suspicious regions that could potentially be overlooked by the radiologist. Also, CADe can be used to confirm radiologist decision about a specific suspicious area. The second type is computer-aided diagnosis (CADx) systems developed to be an aid for the radiologist in better identification of abnormality type (i.e. benign, malignant, etc.).

\section{LITERATURE REVIEW}

Many studies were done to improve CAD system accuracy of early detection and diagnose the abnormalities in women breast tissues. R. Nithya et. al. (2011), proposed a CAD system to classify abnormal vs. normal tissue of mammogram images. A total of 250 images were collected from digital mammogram database (DDSM). Gray Level co-occurrence matrix (GLCM) features were calculated for 4 different directions or angles $\left(0^{\circ}, 45^{\circ}, 90^{\circ}, 145^{\circ}\right)$ in 4 different distances $(1,2,3,4)$. In the study, five features were calculated under GLCM (energy, entropy, correlation, sum of square variance and homogeneity). A neural network was used for classification, divided to three layers (input, output, and hidden layer), resulting 96\% accuracy, 100\% sensitivity, and $96 \%$ specificity.

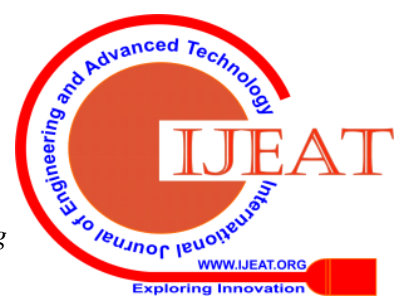




\section{Computer-Aided Diagnosis of Mammography Cancer}

Recorded results led to better performance in CADe. Statistical features with feature selection method could have improved overall accuracy of the system [6].

Rajkumar K.K, G. Raju et. al. (2014), proposed a CADx system to Detect and Classify the abnormal mammogram images using Lazy Classifiers. Multi-stage classification method applied. The first stage is to apply a set of GLCM features applied and extracted from multiple region of interest (ROIs: 8x8, 16x16, 32x32) taken form mini-MIAS database. Then, the images classified to normal and abnormal using lazy classifiers (instance-based classifier $\left(\mathrm{K}^{*}\right)$, Instance-based Learning (IBL), Locally Weighted Learning (LWL)). After that, all abnormal images resulted from the first stage classified to its illness type depending upon architectural as well as texture patterns found in the image ROI. First stage classifier best result obtained on 32x32 pixel ROI with accuracy $92.40 \%$ and $86.18 \%$ for the second stage classification. It was noticeable that better accuracy obtained in the first and second stage corresponding with increment of ROI size for the three kind of lazy classifiers [8].

Mohamed E. Elmanna, et. al. (2015), proposed CADx system for classify masses in mammogram images. Images have been collected from the digital database for screening mammography (DDSM) with resolution of 50 micron and grayscale level of 12-bit. Images down sampled by 0.25 by nearest- neighbor interpolation. The ROI taken manually from the image in window of 32x32 pixels. Tao Wu technique applied for ROI enhancement. Fifty-nine (59) features were used to characterize the normal and abnormal images such as first order statistical parameters, wavelet decomposition and GLCM features. Sequential floating forward selection (SFFS) and sequential forward selection (SFS) used for feature selection. Then multiple classifiers were used to classify the images. Best result obtained by KNN classifier with $95.36 \%$ Accuracy, 98\% specificity, and 96\% sensitivity. Recorded results proved the approached CAD was practical for application. It was noticeable that SFFS provided excellent results and showed that SFFS technique can extract the useful features better than SFS [9].

Ancy C A, et. al. (2017), proposed CADe for detection of tumor in mammograms using SVM. Images collected from University of South Florida Digital Mammography (USFDM) databases. A total of 100 pair of images were included for testing the efficiency of the method. Starting with Image denoising using curvelet transform to remove unnecessary noise from selected images. Then contrast enhancement with brightness preserving using recursive mean separate histogram equalization [RMSHE] followed by median filtering and gray level thresholding to perform the morphological segmentation. Five GLCM texture features were extracted. Then Linear SVM is used to classify whether the segmented tissue from image is tumor or not. The accuracy of the method reached $81 \%$ with $73 \%$ sensitivity and 99\% specificity. Specificity rate reveals that GLCM based SVM technique can give better classification results with easy implementations [10].

R.D. Ghongade et. al. (2017), proposed CADx system for breast cancer using random forest (RF) Classifier. Mammogram images with 1024x1024 pixels were used from MIAS database. First, gaussian filter was applied to denoise and smooth the selected images. Then histogram equalization was used to enhance the images contrast. Segmentation is done using region-based method to enhance masses from image background. Otsu's method was applied to execute clustering-based thresholding and then image normalization process done by applying a multiplication of binary mask with the original image. GLCM was used for texture features extraction. Fast Correlation Based Feature Selection (FCBF) method applied to choose the useful features. Image classification is done by Random Forest algorithm to classify the normal, benign and malignant images. The method has achieved $97.32 \%$ accuracy, $97.45 \%$ sensitivity and $98.13 \%$ specificity. The study result showed that RF classifier provides good classification accuracy by decreasing the false positives (FPs) and false negatives (FNs) depending upon the features selection optimization[11].

R. Vijayarajeswari et. al. (2019), proposed CADe to classify mammogram images using SVM classifier and Hough transform. The study focused on fatty-glandular breast cases excluding dense ones. 95 Images collected from Mini-MIAS database to include normal, benign and malignant types. All unwanted information and background noise were removed and pectoral muscle segmentation step occurred in preprocessing stage. Intensity features (entropy, variance, mean and standard deviation) after Hough transform applied on the processed images. The accuracy of classification reaches $94 \%$, obtained by SVM classifier. The study focused on intensity features like mean, variance and entropy can improve the results. The week point in this study that this study only focused on fatty-glandular breast images excluding dense images, while other study usually include more type of images for more applicable results [12].

This study relied on the methodologies of previous studies to develop CADe system. Where, this study focused to use effective preprocessing technique, multiple types of useful features and effective classification techniques to provide accurate results and highlight the most effective features that contributed to improve CADe system for women breast tissues.

\section{METHADOLOGY}

This section will describe the methodology of the research step by step to build up proposed computer aided detection (CADe) algorithm. Building CADe system require 5 main steps as shown in figure 2.1. The software used in this study to build the proposed CADe is MATLAB R2015a
Published By:

Blue Eyes Intelligence Engineering \& Sciences Publication (C) Copyright: All rights reserved. 


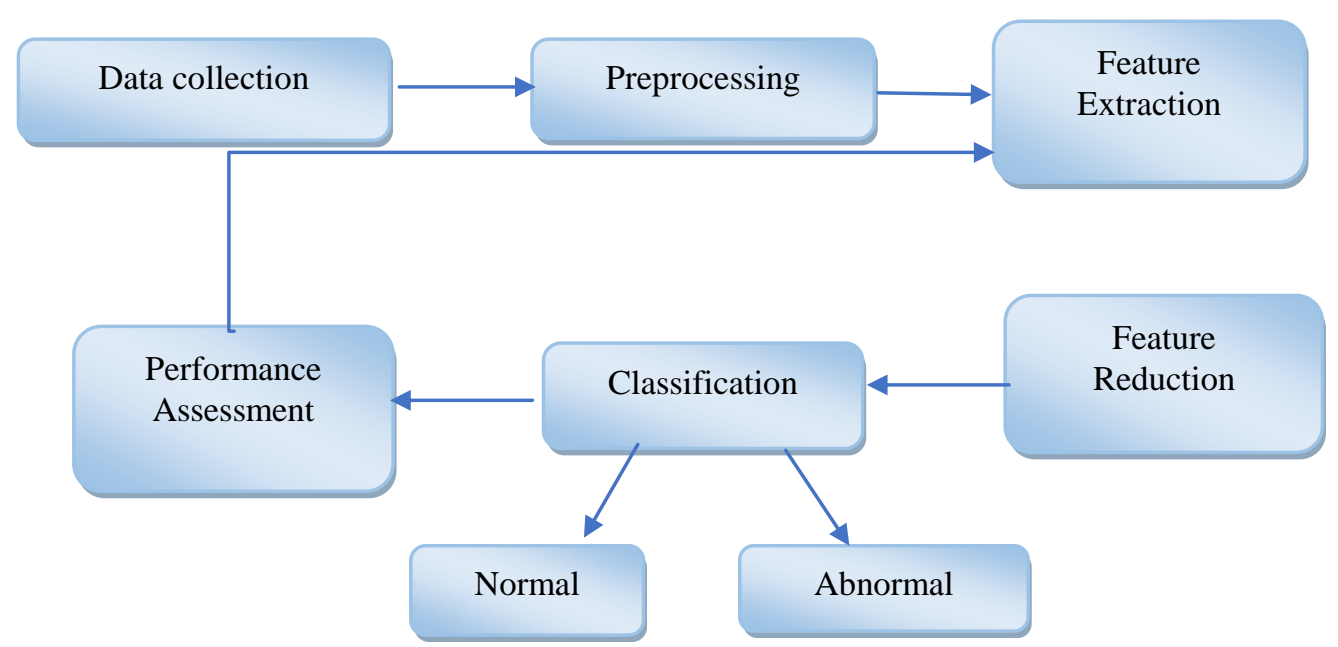

Figure 3.1. (A diagram represent the research CADe algorithm design).

\subsection{Data collection:}

The MIAS database were used through this study. The free database contains digitized images at 50-micron pixel edge and every image is $1024 \times 1024$ pixels[13]. The database contains of 322 images, divided to three different types of cases (208 normal, 51 malignant and 63 benign) mammograms. 230 out of the 322 images are used here, including 115 normal and 115 abnormal images. All 6 types of abnormalities were included in this study (Asymmetry, Architectural distortion, Well-defined masses, ill-defined masses, Speculated masses and Calcification). Images were divided into two groups: 154 images (77 normal \& 77 abnormal) as learning set to train classifiers and 76 images (38 normal \& abnormal) to test the performance of classifiers.

\subsection{Preprocessing:}

\subsubsection{Region of Interest (ROI):}

Three ROIs were cropped manually one by one from each full image of sizes: 32x32, 42x42 \& 52x52 pixels to study each with our algorithm to find the best size in terms of performance, and to test the consistency of results.

\subsubsection{Image Enhancement:}

Images enhancement technique by Tao Wu et al.[14] used to denoise and enhance the contrast between different tissues which will lead to better image visibility. The concept of this technique is to estimate the normalized thickness profile (NTP) of a breast from a mammogram image and enhance the interested area. Preprocessing step was proven as mentioned in previous literature reviews that it leads to more effective result of overall CADe system. The method algorithm of Tao $\mathrm{Wu}$ is described as the following:

a. Background image segmentation by Otsu thresholding

b. Generate blurred images

c. Multiply the segmented images (SI) with blurred image (BI) to make all pixel outside breast region equal to zero.

d. normalize thickness profile (NTP) of BI by applying multiple multi-threshold segmentation technique.

The method uses 5 threshold values $\left(T_{n}\right)$ calculated by the following equation:

$$
T n=\text { Iave } F n
$$

Where $\mathrm{I}_{\text {ave }}$ is the average intensity of (BI) and $F_{n}=(1.2,1.1$, 1.0, 0.9, 0.8). Each threshold of $T_{n}$, the BI was rescaled to get new pixel value $\mathrm{V}$ reset to:

$$
V=\left\{\begin{aligned}
\frac{V}{T n}, & V \leq T n \\
1, & \text { otherwise }
\end{aligned}\right.
$$

NTP obtained by calculating average of the five rescaled images. In figure 2.3 represent a summary of Tao Wu image enhancement technique steps.

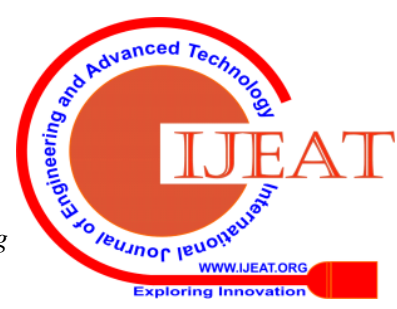



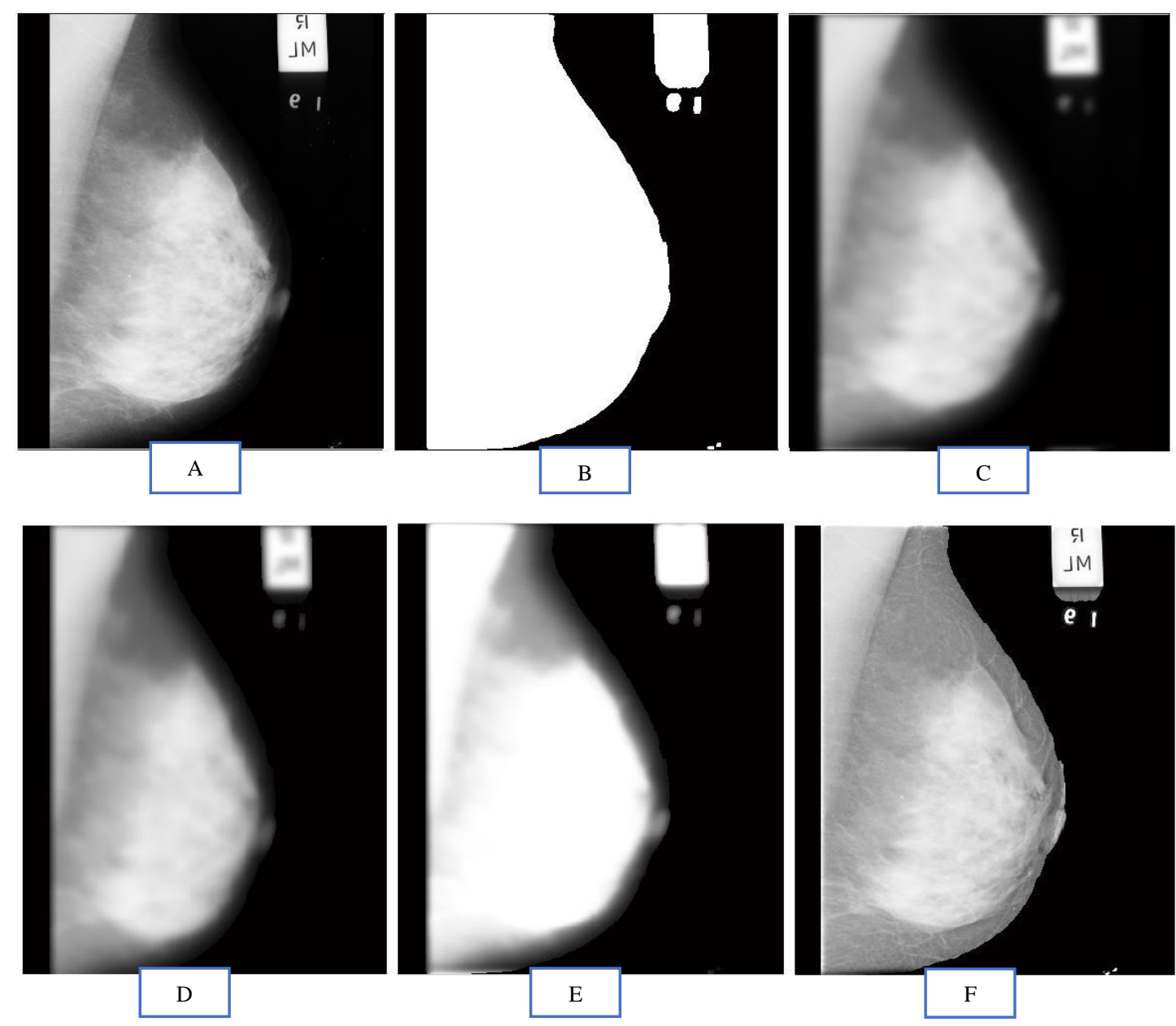

Figure 3.3. (Tao Wu image enhancement technique steps: (A) Original image, (B) Otsu thresholding SI, (C) BI , (D) BI after multiply with SI, (E) NTP image, (F) PE image).

\subsection{Feature Extraction:}

This step plays a main factor in CAD performance. the extracted features act as the mathematical description of characteristics for classifiers to be able to distinguish normal from abnormal lesions. The features extracted from ROI as follows:

- Statistical and shape features on ROI. Those features are: (Mean, STD, Square of STD, Mode, Median, Quantile, Percintile, Third Moment, Entropy, Skewness, kurtosis, Variance, Smoothness)

- GLCM features with level of 32 and shift or distance $(\mathrm{d}=$ 1, 2 and 3) in all direction (angles $=0^{\circ}, 45^{\circ}, 90^{\circ}$ and $135^{\circ}$ ). Those features are: (Contrast, Energy, Correlation, Homogeneity, Entropy, Third Moment, Skewness, kurtosis, Variance)

- Histogram features with two no. of levels (16 and 32)

- Same Statistical features and GLCM features computed from different transformed ROI domains: two types of Daubechies wavelet decomposition domain (db1, db4).

The statistical features were calculated after Daubechies wavelet decomposition transform for details coefficients matrices $\mathrm{HH}, \mathrm{LH}$ and $\mathrm{HL}$ (vertical, and diagonal, respectively) from the matrix using the wavelet Daubechies (db1 and db4) and HL (db1) coefficient matrix used for GLCM features.

\subsection{Feature Selection:}

The total number of features extracted with histogram of level 16 was 462 features, and with level 32 was 574. After that, Sequential Forward Selection (SFS) method were used to reduce the number of features and to find the best useful feature set that result best outcome for each classifier. SFS technique is one of the simplest and probably fastest feature selection algorithms, where features are added incrementally while watching classifier performance to decide whether each feature is contributing to improve overall accuracy or not.

\subsection{Classification:}

This process contains two main phases, learning and testing phases. The features that were selected from the previous stage will be used in the classification stage. In the learning phase, known data represent clearly the nature of the lesion whether it is a normal or abnormal, to teach and train the classifier. In the testing phase, classification was done using trained structure of classifier. In this study SFS were used with five classifiers (level 3 and 5 K-voting Nearest Neighbor (KNN) classifiers, and three SVM classifiers of different kernel (Linear, Polynomial and MLP). 


\section{RESULTS \& DISSCUSSION}

Results were obtained by using a test ROI set of 76 images (38 normal \& 38 abnormal). Then, 5 parameters (PPV, NPV, Sensitivity, Specificity and Accuracy) used to evaluate the performance of each classifier. Following tables and figure show the study best results by presenting the performance of each classifier with highlight on useful features by using SFS as feature reduction technique.

Table 4.1 Present overall CADe performance evaluation Results of 32x32 ROIs with Histogram level=32 (in \%).

\begin{tabular}{|l|c|c|l|l|l|}
\hline & \multicolumn{2}{|c|}{ KNN } & \multicolumn{3}{c|}{ SVM } \\
\hline & K=3 & K=5 & Linear & Polynomial & Mlp \\
\hline ERROR & 15.7 & 14.4 & 02.63 & 06.58 & 22.37 \\
\hline RATE & 9 & 7 & & & \\
\hline Accuracy & 84.2 & 85.5 & 97.37 & 93.42 & 77.63 \\
\hline Sensitivity & $\begin{array}{c}82.5 \\
0\end{array}$ & $\begin{array}{c}81.4 \\
0\end{array}$ & 95.00 & 97.14 & 73.33 \\
\hline Specificity & $\begin{array}{c}86.1 \\
1\end{array}$ & $\begin{array}{c}90.9 \\
1\end{array}$ & 100 & 90.24 & 83.87 \\
\hline PPV & 86.8 & 92.1 & 100 & 89.47 & 86.84 \\
\hline NPV & 81.5 & 78.9 & 94.74 & 97.37 & 68.42 \\
\hline AUC & 84.2 & 85.5 & 97.37 & 93.42 & 77.63 \\
\hline $\begin{array}{l}\text { No. of } \\
\text { Features }\end{array}$ & 5 & 8 & 7 & 5 & 3 \\
\hline
\end{tabular}

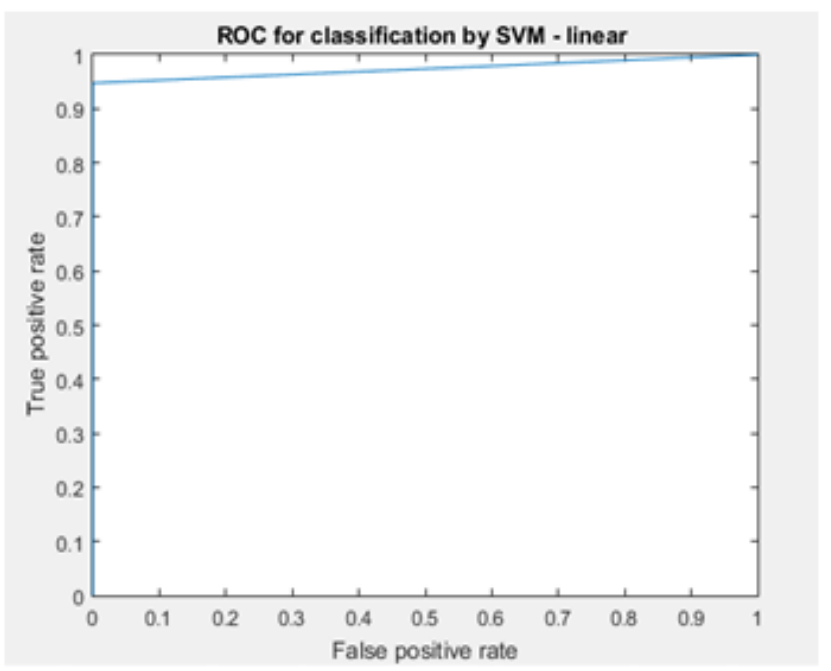

Figure 4.1 Present the ROC of Linear SVM classifier of 32x32 ROIs with Histogram level=32.
Table 4.2 Present overall CADe performance evaluation Results of 42x42 ROIs with Histogram level $=16$ (in \%). KNN SVM

\begin{tabular}{|c|c|c|c|c|c|}
\hline & \multicolumn{2}{|c|}{ KNN } & \multicolumn{3}{|c|}{ SVM } \\
\hline & $\mathrm{K}=3$ & $\mathrm{~K}=5$ & Linear & Polynomial & Mlp \\
\hline $\begin{array}{l}\text { ERROR } \\
\text { RATE }\end{array}$ & $\begin{array}{c}19.7 \\
4\end{array}$ & $\begin{array}{c}17.1 \\
1\end{array}$ & 06.58 & 75.00 & $\begin{array}{c}14.4 \\
7\end{array}$ \\
\hline Accuracy & $\begin{array}{c}80.2 \\
6\end{array}$ & $\begin{array}{c}82.8 \\
9\end{array}$ & 93.42 & 25.00 & $\begin{array}{c}85.5 \\
3\end{array}$ \\
\hline Sensitivity & $\begin{array}{c}81.0 \\
8\end{array}$ & $\begin{array}{c}79.0 \\
7\end{array}$ & 97.14 & 17.24 & $\begin{array}{c}80.0 \\
0\end{array}$ \\
\hline Specificity & $\begin{array}{c}79.4 \\
9\end{array}$ & $\begin{array}{c}87.8 \\
8\end{array}$ & 90.24 & 29.79 & $\begin{array}{c}93.5 \\
5\end{array}$ \\
\hline PPV & $\begin{array}{c}78.9 \\
5\end{array}$ & $\begin{array}{c}89.4 \\
7\end{array}$ & 89.47 & 13.16 & $\begin{array}{c}94.7 \\
4\end{array}$ \\
\hline NPV & $\begin{array}{c}81.5 \\
8\end{array}$ & $\begin{array}{c}76.3 \\
2\end{array}$ & 97.37 & 36.84 & $\begin{array}{c}76.3 \\
2\end{array}$ \\
\hline AUC & $\begin{array}{c}80.2 \\
6\end{array}$ & $\begin{array}{c}82.8 \\
9\end{array}$ & 93.42 & 25.00 & $\begin{array}{c}85.5 \\
3\end{array}$ \\
\hline $\begin{array}{l}\text { No.of } \\
\text { Features }\end{array}$ & 6 & 7 & 4 & 4 & 3 \\
\hline
\end{tabular}

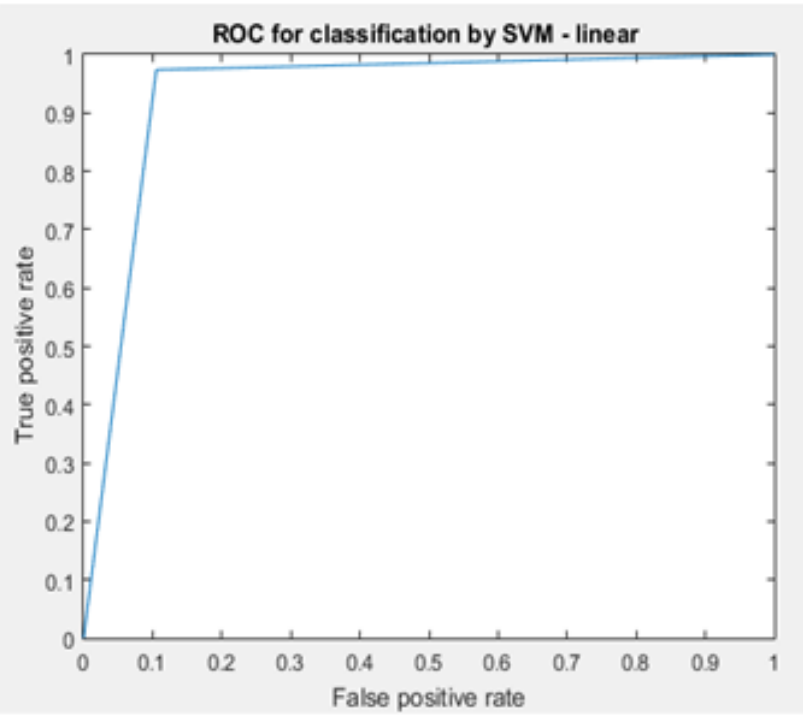

Figure 4.2 Present the ROC of Linear SVM classifier of 42x42 ROIs with Histogram level=16.

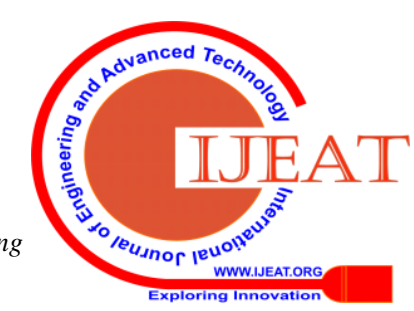




\section{Computer-Aided Diagnosis of Mammography Cancer}

Table 4.3: Present overall CADe performance evaluation Results of 52x52 ROIs with Histogram level =32 (in \%)

\begin{tabular}{|c|c|c|c|c|c|}
\hline & \multicolumn{2}{|c|}{ KNN } & \multicolumn{3}{c|}{ SVM } \\
\hline & $\mathrm{K}=3$ & $\mathrm{~K}=5$ & Linear & Polynomial & Mlp \\
\hline $\begin{array}{c}\text { ERROR } \\
\text { RATE }\end{array}$ & $\begin{array}{c}22.3 \\
7\end{array}$ & $\begin{array}{c}23.6 \\
8\end{array}$ & 05.26 & 19.74 & $\begin{array}{c}14.4 \\
7\end{array}$ \\
\hline Accuracy & $\begin{array}{c}77.6 \\
3\end{array}$ & $\begin{array}{c}76.3 \\
2\end{array}$ & 94.74 & 80.26 & $\begin{array}{c}85.5 \\
3\end{array}$ \\
\hline Sensitivity & $\begin{array}{c}76.9 \\
2\end{array}$ & $\begin{array}{c}73.8 \\
1\end{array}$ & 97.22 & 79.49 & $\begin{array}{c}88.5 \\
7\end{array}$ \\
\hline Specificity & $\begin{array}{c}78.3 \\
8\end{array}$ & $\begin{array}{c}79.4 \\
1\end{array}$ & 92.50 & 81.08 & $\begin{array}{c}82.9 \\
3\end{array}$ \\
\hline PPV & $\begin{array}{c}78.9 \\
5\end{array}$ & $\begin{array}{c}81.5 \\
8\end{array}$ & 92.11 & 81.58 & $\begin{array}{c}81.5 \\
8\end{array}$ \\
\hline NPV & $\begin{array}{c}76.3 \\
2\end{array}$ & $\begin{array}{c}71.0 \\
5\end{array}$ & 97.37 & 78.95 & $\begin{array}{c}89.4 \\
7\end{array}$ \\
\hline AUC & $\begin{array}{c}77.6 \\
3\end{array}$ & $\begin{array}{c}76.3 \\
2\end{array}$ & 94.74 & 80.26 & $\begin{array}{c}85.5 \\
3\end{array}$ \\
\hline $\begin{array}{c}\text { No. of } \\
\text { Features }\end{array}$ & 2 & 7 & 6 & 3 & 5 \\
\hline
\end{tabular}

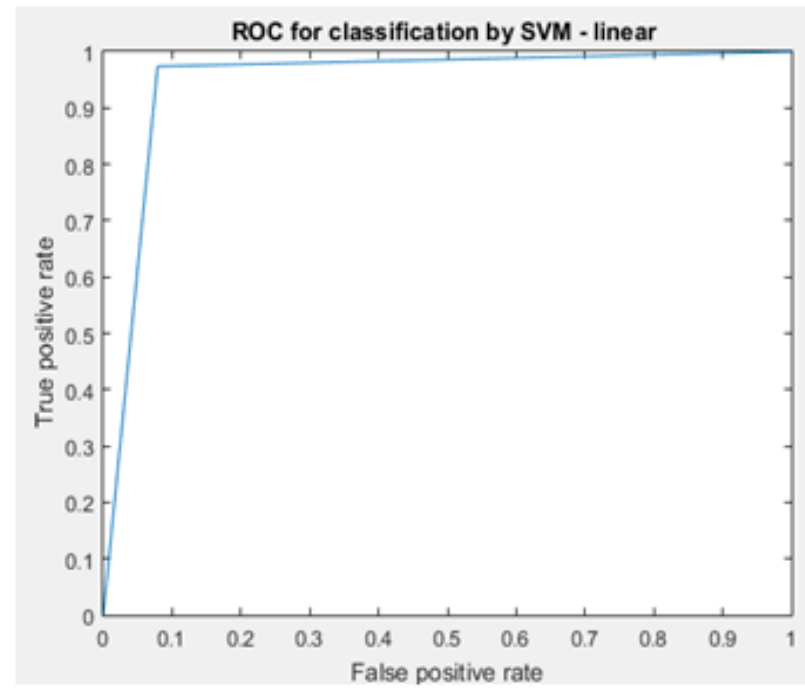

Figure 4.3 Present the ROC of Linear SVM classifier of 52x52 ROIs with Histogram level=32.

Previous results with different image sizes showed promising performance. Comparing between classifiers performances, it was noticeable that SVM linear had the highest performance. SVM linear has the best performance reached with ROI size 32x32 pixels while using histogram level $=32$. Recorded results were: accuracy $=97.37 \%$, Sensitivity $=95 \%$, Specify $=100 \%$, PPV $=100 \%$, NPV $=94.74 \%$. This result reached using 7 features only after SFS. It was noticeable that better results achieved in general while using histogram with level $=32$ in all ROI sizes. Also, good stability in results were remarkable with different image sizes which can tell that the used methodology in this study is reproducible. The results also indicate some useful features are well-representing to abnormalities across different classifiers such as: Mean, STD, Square of STD, Mode, Median, Quantile (10\%), Quantile (70\%), Quantile (90\%),
Percentile (30\%), throughout multiple histogram levels under spatial and DWT spaces.

\section{CONCLUSION}

The study introduces a developed CADe system to support radiologists in better detection of abnormalities in mammogram images including microcalcification to support early detection of cancer. MIAS database were used to propose CADe system. 3 different sizes of ROIs were prepared to compare and test the stability of proposed CADe system. Good performance stability of the system observed with Linear SVM classifier with different ROIs. Best result was obtained with ROI of 32x32 pixel. Accuracy of system reached $97.37 \%$ with $100 \%$ Specificity and 95\% Sensitivity. The result was better from many previous studies and competitive to many other. Using a huge number of different features support the CADe system to be picky enough to

separate normal from abnormal images of 6 different types of abnormalities. Many useful features well-fitting to data across different classifiers such as: Mean, STD, Square of STD, Mode, Median, Quantile (10\%), Quantile (70\%), Quantile (90\%), Percentile (30\%), throughout multiple histogram levels under spatial and DWT spaces.

\section{REFERENCES}

1. Tiedeu, A., et al., Texture-based analysis of clustered microcalcifications detected on mammograms. Digital Signal Processing, 2012. 22(1): p. 124-132.

2. Christoyianni, I., et al., Computer aided diagnosis of breast cancer in digitized mammograms. Computerized Medical Imaging and Graphics, 2002. 26(5): p. 309-319.

3. Christoyianni, I., et al., Breast tissue classification in mammograms using ICA mixture models. Artificial Neural Networks-Icann 2001, Proceedings, 2001. 2130: p. 554-560.

4. Jirari, M. and Ieee. A computer aided detection system for digital mammograms based on radial basis functions and feature extraction techniques. in 27th Annual International Conference of the IEEE-Engineering-in-Medicine-and-Biology-Society. 2005. Shanghai, PEOPLES R CHINA: Ieee.

5. Alolfe, M.A., et al., COMPUTER AIDED DIAGNOSIS IN DIGITAL MAMMOGRAPHY USING COMBINED SUPPORT VECTOR MACHINE AND LINEAR DISCRIMINANT ANALYASIS CLASSIFICATION. 2009 16th Ieee International Conference on Image Processing, Vols 1-6, 2009: p. 2609-2612.

6. Nithya, R., Classification of Normal and Abnormal Patterns in Digital Mammograms for Diagnosis of Breast Cancer. 2011. 28(6).

7. M.S.Elbailly, K8. Hand-Held Computer Aided Diagnostic System with Application in Mammography. 2013: p. 16-18.

8. K.K, D.R., Detecting and Classification of Abnormal Mammogram Images Using Lazy Classifiers. International Journal of Engineering and Technical Research, 2014. 2(10): p. 2321-0869.

9. Elmanna, M.E., Y.M. Kadah, and Ieee, Implementation of Practical Computer Aided Diagnosis System for Classification of Masses in Digital Mammograms. 2015 International Conference on Computing, Control, Networking, Electronics and Embedded Systems Engineering (ICCNEEE), 2015: p. 336-341.

10. Ancy, C.A., L.S. Nair, and Ieee, An Efficient CAD for Detection of Tumour in Mammograms using SVM. 2017 International Conference on Communication and Signal Processing (Iccsp), 2017: p. 1431-1435.

11. Ghongade, R.D., D.G. Wakde, and Ieee, Computer-aided Diagnosis System for Breast Cancer Using RF Classifier. 2017 2nd Ieee International Conference on Wireless Communications, Signal Processing and Networking (Wispnet), 2017: p. 1068-1072.

Published By:

Blue Eyes Intelligence Engineering \& Sciences Publication

(C) Copyright: All rights reserved. 
12. Vijayarajeswari, R., et al., Classification of mammogram for early detection of breast cancer using SVM classifier and Hough transform. Measurement, 2019. 146: p. 800-805.

13. The Mini-MIAS database. 2019; Available from: http://peipa.essex.ac.uk/info/mias.html.

14. Wu, T., Multi-threshold peripheral equalization method and apparatus for digital mammography and breast tomosynthesis. 2010.

15. Osman, Y., Breast Cancer Computer-Aided Detection System based on Simple Statistical Features and SVM Classification. Internationl Journal of Advanced Computer Science and Application, 2020. 11: p. 9.

16. Al-antari, M.A.S., Computer-Aided Breast Cancer Detection and Diagnosis from Digital Mammograms, in Biomedical Engineering Department. 2015, Faculty of Engineering , Cairo University: Cairo.

17. Elmanna, M.E.M., COMPUTER AIDED DIAGNOSIS SYSTEM FOR DIGITAL MAMMOGRAPHY, in System and Biomedical Engineering at Faculty of Engineering. 2013, Cairo University: Cairo. p. 90.

18. Alam, N. and R. Zwiggelaar, Automatic classification of clustered microcalcifications in digitized mammogram using ensemble learning, in 14th International Workshop on Breast Imaging, E.A. Krupinski, Editor. 2018, Spie-Int Soc Optical Engineering: Bellingham.

19. AlQoud, A. and M.A. Jaffar, Hybrid Gabor based Local Binary Patterns Texture Features for classification of Breast Mammograms. International Journal of Computer Science and Network Security, 2016. 16(4): p. 16-21.

20. Chayakulkheeree, J., et al., Phylloides tumor of the breast containing osseous, chondroid, and rhabdomyomatous differentiation with osteosarcomatous pulmonary metastases. Asian Biomedicine, 2009. 3(3): p. 293-297.

21. Muttarak, M., P. Kongmebhol, and N. Sukhamwang, Breast calcifications: which are malignant? Singapore Medical Journal, 2009. 50(9): p. 907-913.

22. Pataki, B. and L. Lasztovicza, Extending Mammographic Microcalcification Detection Method to Cluster Characterization, in 4th European Conference of the International Federation for Medical and Biological Engineering, J. VanderSloten, et al., Editors. 2009, Springer: New York. p. 604-607.

23. Shen, L., R.M. Rangayyan, and J.E.L. Desautels, APPLICATION OF SHAPE-ANALYSIS TO MAMMOGRAPHIC CALCIFICATIONS. Ieee Transactions on Medical Imaging, 1994. 13(2): p. 263-274.

24. Suzuki, K., A. Shiraishi, and A. Arakawa, Analysis of stereotactic vacuum-assisted breast biopsy for patients with segmental calcifications. Japanese Journal of Radiology, 2009. 27(10): p. 450-454.

25. Wei, L.Y., et al., A study of several CAD methods for classification of clustered microcalcifications, in Medical Imaging 2005: Image Processing, Pt 1-3, J.M. Fitzpatrick and J.M. Reinhardt, Editors. 2005, Spie-Int Soc Optical Engineering: Bellingham. p. 1-8.

26. Wei, L.Y., et al., A study on several machine-learning methods for classification of malignant and benign clustered microcalcifications. Ieee Transactions on Medical Imaging, 2005. 24(3): p. 371-380.

27. Lee, S.K., et al., A computer-aided design mammography screening system for detection and classification of microcalcifications. International Journal of Medical Informatics, 2000. 60(1): p. 29-57.

28. Bottema, M.J. and J.P. Slavotinek, Detection and classification of lobular and DCIS (small cell) microcalcifications in digital mammograms. Pattern Recognition Letters, 2000. 21(13-14): p. 1209-1214.

29. Khehra, B.S. and A.P.S. Pharwaha, Classification of Clustered Microcalcifications using MLFFBP-ANN and SVM. Egyptian Informatics Journal, 2016. 17(1): p. 11-20.

30. Yu, S.N. and Y.K. Huang, Detection of microcalcifications in digital mammograms using combined model-based and statistical textural features. Expert Systems with Applications, 2010. 37(7): p. 5461-5469.

31. Lee, S.K., et al., Classification of clustered microcalcifications using a Shape Cognitron neural network. Neural Networks, 2003. 16(1): p. 121-132.

32. Cheng, H.D., et al., Approaches for automated detection and classification of masses in mammograms. Pattern Recognition, 2006. 39(4): p. 646-668.

33. Sultana, A., et al., Detection of Mammographic Microcalcifications Using a Refined Region-Growing Approach. Isscs 2009: International Symposium on Signals, Circuits and Systems, Vols 1 and 2 , Proceedings, 2009: p. 229-232.

34. Rizzi, M., M. D'Aloia, and B. Castagnolo, Review: Health Care CAD Systems for Breast Microcalcification Cluster Detection. Journal of Medical and Biological Engineering, 2012. 32(3): p. 147-156.

35. Al-antari, M.A., et al., A fully integrated computer-aided diagnosis system for digital $X$-ray mammograms via deep learning detection,

Published By:

Blue Eyes Intelligence Engineering \& Sciences Publication
Informatics, 2018. 117: p. 44-54.

36. Mehdi, M.Z., et al., An efficient technique for the extraction of microcalcification's severity features. 2016 Second International Image Processing, Applications and Systems. 2016, New York: Ieee.

37. Alolfe, M.A., et al. COMPUTER-AIDED DIAGNOSTIC SYSTEM BASED ON WAVELET ANALYSIS FOR MICROCALCIFICATION DETECTION IN DIGITAL MAMMOGRAMS. in Cairo International Biomedical Engineering Conference. 2008. Cairo, EGYPT: Ieee.

38. Alolfe, M.A., et al. Feature Selection in Computer Aided Diagnostic System for Microcalcification Detection in Digital Mammograms. in 26th National Radio Science Conference (NRSC 2009). 2009. Future Univ, Fac Engn, Cairo, EGYPT: Ieee.

39. Singh, B. and M. Kaur, An approach for classification of malignant and benign microcalcification clusters. Sadhana-Academy Proceedings in Engineering Sciences, 2018. 43(3): p. 18.

40. El-Sokary, N., et al., Computer-Aided Detection System for Breast Cancer Based on GMM and SVM. Arab Journal of Nuclear Sciences and Applications, 2019. 52(2): p. 142-150.

41. Liang, C.X., et al., A computer-aided diagnosis scheme of breast lesion classification using GLGLM and shape features: Combined-view and multi-classifiers. Physica Medica-European Journal of Medical Physics, 2018. 55: p. 61-72.

42. Kumar, S. and M. Chandra, Detection of Microcalcification Using the Wavelet Based Adaptive Sigmoid Function and Neural Network. Journal of Information Processing Systems, 2017. 13(4): p. 703-715.

43. Raghavendra, U., et al., Application of Gabor wavelet and Locality Sensitive Discriminant Analysis for automated identification of breast cancer using digitized mammogram images. Applied Soft Computing, 2016. 46: p. 151-161.

44. Pereira, D.C., R.P. Ramos, and M.Z. do Nascimento, Segmentation and detection of breast cancer in mammograms combining wavelet analysis and genetic algorithm. Computer Methods and Programs in Biomedicine, 2014. 114(1): p. 14.

45. do Nascimento, M.Z., et al., Classification of masses in mammographic image using wavelet domain features and polynomial classifier. Expert Systems with Applications, 2013. 40(15): p. 6213-6221.

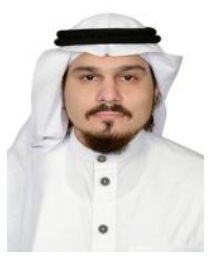

\section{AUTHORS PROFILE}

Eng. Loai S. Kinani Graduate Student of M.Sc Program of Biomedical Engineering at King Abdulaziz University, Jeddah, Saudi Arabia. Bachelor degree in Biomedical Engineering from King Abdulaziz University.

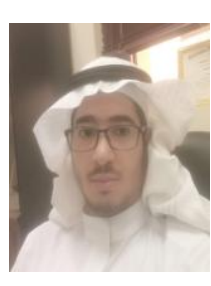

Dr. Umar S. Alqasemi, Associate Professor of Biomedical Engineering at the Dept. of Electrical and Computer Engineering, King, Abdulaziz University, Jeddah 21589, Saudi Arabia. PhD and MSc degree in Biomedical Engineering from UConn, Storrs, USA Research work in ultrasound, optical, and photoacoustic imaging, medical imaging recognition, bioelectronics, and digital and analog signal and image processing 